\title{
Smooth compactness of self-shrinkers
}

\author{
Tobias H. Colding and William P. Minicozzi II*
}

\begin{abstract}
We prove a smooth compactness theorem for the space of embedded self-shrinkers in $\mathbb{R}^{3}$. Since self-shrinkers model singularities in mean curvature flow, this theorem can be thought of as a compactness result for the space of all singularities and it plays an important role in studying generic mean curvature flow.
\end{abstract}

Mathematics Subject Classification (2010). 53C44.

Keywords. Geometric flows, mean curvature flow, self-shrinker, singularities.

\section{Introduction}

A surface $\Sigma \subset \mathbb{R}^{3}$ is said to be a self-shrinker if it satisfies

$$
H=\frac{\langle x, \boldsymbol{n}\rangle}{2},
$$

where $H=\operatorname{div} \boldsymbol{n}$ is the mean curvature, $x$ is the position vector, and $\boldsymbol{n}$ is the unit normal. This is easily seen to be equivalent to that $\Sigma$ is the $t=-1$ time-slice $^{1}$ of a mean curvature flow ("MCF") moving by rescalings, i.e., where the time $t$ slice is given by $\sqrt{-t} \Sigma$.

Self-shrinkers play an important role in the study of mean curvature flow. Not only are they the simplest examples (those where later time slices are rescalings of earlier), but they also describe all possible blow ups at a given singularity of a mean curvature flow. The idea is that we can rescale a MCF in space and time to obtain a new MCF, thereby expanding a neighborhood of the point that we want to focus on. Huisken's monotonicity, [H3], and Ilmanen's compactness Theorem, [I1], give a subsequence converging to a limiting solution of the MCF; cf. [I1], [W1]. This limit, which is called a tangent flow, achieves equality in Huisken's monotonicity and, thus, its time $t$ slice is $\sqrt{-t} \Sigma$ where $\Sigma$ is a self-shrinker.

The main result of this paper is the following smooth compactness theorem for self-shrinkers in $\mathbb{R}^{3}$ that is used in [CM1].

\footnotetext{
*The authors were partially supported by NSF Grants DMS 0606629 and DMS 0405695.

${ }^{1}$ In [H3], self-shrinkers are time $t=-\frac{1}{2}$ slices of self-shrinking MCFs; these satisfy $H=\langle x, \boldsymbol{n}\rangle$.
} 
Theorem 0.2. Given an integer $g \geq 0$ and a constant $V>0$, the space of smooth complete embedded self-shrinkers $\Sigma \subset \mathbb{R}^{3}$ with

- genus at most g,

- $\partial \Sigma=\emptyset$,

- Area $\left(B_{R}\left(x_{0}\right) \cap \Sigma\right) \leq V R^{2}$ for all $x_{0} \in \mathbb{R}^{3}$ and all $R>0$

is compact.

Namely, any sequence of these has a subsequence that converges in the topology of $C^{m}$ convergence on compact subsets for any $m \geq 2$.

The surfaces in this theorem are assumed to be homeomorphic to closed surfaces with finitely many disjoint disks removed. The genus of the surface is defined to be the genus of the corresponding closed surface. For example, an annulus is a sphere with two disks removed and, thus, has genus zero. Below, we will use that the genus is monotone in the sense that if $\Sigma_{1} \subset \Sigma_{2}$, then the genus of $\Sigma_{1}$ is at most that of $\Sigma_{2}$.

As mentioned, the main motivation for this result is that self-shrinkers model singularities in mean curvature flow. Thus, the above theorem can be thought of as a compactness result for the space of all singularities. In practice, scale-invariant local area bound, smoothness, and the genus bound will automatically come from corresponding bounds on the initial surface in a MCF. Namely:

- Area bounds are a direct consequence of Huisken's monotonicity formula, [H3]. ${ }^{2}$

- Ilmanen proved that in $\mathbb{R}^{3}$ tangent flows at the first singular time must be smooth and have genus at most that of the initial surface; see Theorem 2 of [I1] and page 21 of [I1], respectively.

Conjecturally, the smoothness and genus bound hold at all singular times:

- Ilmanen conjectured that tangent flows are smooth and have multiplicity one at all singularities. If this conjecture holds, then it would follow from Brakke's regularity theorem that near a singularity the flow can be written as a graph of a function with small gradient over the tangent flow. Combining this with the above mentioned monotonicity of the genus of subsets and a result of White, [W3], asserting that the genus of the evolving surfaces are always bounded by that of the initial surface, we get conjecturally that the genus of the tangent flow is at most that of the initial surface.

Our compactness theorem will play an important role in understanding generic mean curvature flow in [CM1]. Namely, in [CM1], we will see that it follows immediately from compactness together with the classification of (entropy) stable selfsimilar shrinkers proven in [CM1] that given an integer $m$ and $\delta>0$, there exists an $\epsilon=\epsilon(m, \delta, V, g)>0$ such that:

\footnotetext{
${ }^{2} \mathrm{See}$, for instance, Corollary 2.13 in [CM1].
} 
- For any unstable self-similar shrinker in $\mathbb{R}^{3}$ satisfying the assumptions of Theorem 0.2 , there is a surface $\delta$-close to it in the $C^{m}$ topology and with entropy less than that of the self-similar shrinker $-\epsilon$.

This is, in particular, a key to showing that mean curvature flow that disappears in a compact point does so generically in a round point; see [CM1] for details and further applications.

The simplest examples of self-shrinkers in $\mathbb{R}^{3}$ are the plane $\mathbb{R}^{2}$, the sphere of radius 2 , and the cylinder $\mathbb{S}^{1} \times \mathbb{R}^{1}$ (where the $\mathbb{S}^{1}$ has radius $\sqrt{2}$ ). Combining [H3], [H4], and Theorem 0.17 in [CM1] it follows that these are the only smooth embedded self-shrinkers with $H \geq 0$ and polynomial volume growth. ${ }^{3}$ It follows from this that spheres and cylinders are isolated (among all self-shrinkers) in the $C^{2}$-topology. On the other hand, by Brakke's theorem, [Br], any self-shrinker with entropy sufficiently close to one (which is the entropy of the plane) must be flat, so planes are also isolated and we see that all three of the simplest self-shrinkers are isolated. Moreover, one of the key results of [CM1] (see Theorem 0.7 there) was to show that these are the only (entropy) stable self-shrinkers. In sum, if a self-shrinker has $H \geq 0$ or is stable, then it is one of the three simplest types. Moreover, all of these are isolated among all selfshrinkers. ${ }^{4}$ However, there are expected to be many examples of self-shrinkers in $\mathbb{R}^{3}$ where $H$ changes sign or that are unstable. In particular, Angenent, [A], constructed a self-shrinking torus of revolution and there is numerical evidence for a number of other examples; cf. Chopp, [Ch], Angenent-Chopp-Ilmanen, [AChI], Ilmanen, [12], and Nguyen, [N1], [N2]. These examples suggest that compactness fails to hold without a genus bound.

There are three key ingredients in the proof of the compactness theorem. The first is a singular compactness theorem that gives a subsequence that converges to a smooth limit away from a locally finite set of points. Second, we show that if the convergence is not smooth, then the limiting self-shrinker is $L$-stable, where $L$-stable means that for any compactly supported function $u$ we have

$$
\int_{\Sigma}(-u L u) \mathrm{e}^{-\frac{|x|^{2}}{4}} \geq 0 .
$$

Here $L$ is the second order operator from [CM1] that is given by

$$
L u=\Delta u+|A|^{2} u-\frac{1}{2}\langle x, \nabla u\rangle+\frac{1}{2} u .
$$

The last ingredient is the following result from [CM1]:

\footnotetext{
${ }^{3}$ Huisken, [H3], [H4], showed that these are the only smooth embedded self-shrinkers with $H \geq 0,|A|$ bounded, and polynomial volume growth. In [CM1], we prove that this is the case even without assuming a bound on $|A|$.

${ }^{4}$ Both the classification of stable self shrinkers from [CM1] and that those are isolated are implicitly used in the application in [CM1], mentioned above, of our compactness theorem to prove that the $\epsilon>0$ above can be chosen independently of the self-shrinker and not just independently for all self-shrinkers a definite distance away from one of the stable ones.
} 
Theorem 0.5 ([CM1]). There are no L-stable smooth complete self-shrinkers without boundary and with polynomial volume growth in $\mathbb{R}^{n+1}$.

To keep this paper self-contained, we will prove Theorem 0.5 in an appendix.

Finally, we note that the results of [CM4]-[CM8] suggest that there is a compactness theorem for embedded self-shrinkers even without an area bound. However, as mentioned above, then it follows from Huisken's monotonicity formula that selfshrinkers arising as tangent flows at singularities of a MCF starting at a smooth closed surface automatically satisfy an area bound for some constant depending only on the initial surface.

0.1. Conventions and notation. A one-parameter family $M_{t}$ of hypersurfaces in $\mathbb{R}^{n+1}$ flows by mean curvature if

$$
\left(\partial_{t} X\right)^{\perp}=-H \boldsymbol{n},
$$

where $\boldsymbol{n}$ is the outward unit normal and the mean curvature $H$ is given by $H=\operatorname{div} \boldsymbol{n}$. With this convention, $H$ is $n / R$ on the $n$-sphere of radius $R$ in $\mathbb{R}^{n+1}$ and $H$ is $k / R$ on the "cylinder" $\mathbb{S}^{k} \times \mathbb{R}^{n-k} \subset \mathbb{R}^{n+1}$ of radius $R$. If $e_{i}$ is an orthonormal frame for $\Sigma$, the coefficients of the second fundamental form are defined to be $a_{i j}=\left\langle\nabla_{e_{i}} e_{j}, \boldsymbol{n}\right\rangle$. In particular, we have

$$
\nabla_{e_{i}} \boldsymbol{n}=-a_{i j} e_{j} .
$$

Since $\left\langle\nabla_{\boldsymbol{n}} \boldsymbol{n}, \boldsymbol{n}\right\rangle=0$, the mean curvature is $H=\left\langle\nabla_{e_{i}} \boldsymbol{n}, e_{i}\right\rangle=-a_{i i}$ where by convention we are summing over repeated indices.

\section{The self-shrinker equation}

The starting point for understanding self-shrinkers is to realize that there are several other ways to characterize self-shrinkers that are equivalent to the equation (0.1):

(1) The one-parameter family of hypersurfaces $\sqrt{-t} \Sigma \subset \mathbb{R}^{n+1}$ satisfies MCF.

(2) $\Sigma$ is a minimal hypersurface in $\mathbb{R}^{n+1}$, not with the Euclidean metric $\delta_{i j}$, but with the conformally changed metric $g_{i j}=\mathrm{e}^{\frac{-|x|^{2}}{2 n}} \delta_{i j}$.

(3) $\Sigma$ is a critical point for the functional $F$ defined on a hypersurface $\Sigma \subset \mathbb{R}^{n+1}$ by

$$
F(\Sigma)=(4 \pi)^{-n / 2} \int_{\Sigma} \mathrm{e}^{\frac{-|x|^{2}}{4}} d \mu .
$$

The characterization (2) is particularly useful since it will allow us to use local estimates and compactness theorems for minimal surfaces to get corresponding results for self-shrinkers. 
1.1. The equivalence of (1), (2), and (3). The fact that (1), (2), and (3) are equivalent to satisfying the self-shrinker equation (0.1) is well known, but we will include a short proof of this in the next two lemmas.

Lemma 1.2. If a hypersurface $\Sigma$ satisfies (0.1), then $M_{t}=\sqrt{-t} \Sigma$ satisfies MCF and

$$
H_{M_{t}}=-\frac{\left\langle x, \boldsymbol{n}_{M_{t}}\right\rangle}{2 t} .
$$

Conversely, if $M_{t}$ is an MCF, then $M_{t}=\sqrt{-t} M_{-1}$ if and only if $M_{t}$ satisfies (1.3).

Proof. If $\Sigma$ is a hypersurface that satisfies (0.1), then we set $M_{t}=\sqrt{-t} \Sigma$ and $x(p, t)=\sqrt{-t} p$ for $p \in \Sigma$. It follows that $\boldsymbol{n}_{M_{t}}(x(p, t))=\boldsymbol{n}_{\Sigma}(p), H_{M_{t}}(x(p, t))=$ $\frac{H_{\Sigma}(p)}{\sqrt{-t}}$, and $\partial_{t} x=-\frac{p}{2 \sqrt{-t}}$. Thus, $\left(\partial_{t} x\right)^{\perp}=-\frac{\langle p, \boldsymbol{n}\rangle}{2 \sqrt{-t}}=-H_{M_{t}}(x(p, t))$. This proves that $M_{t}$ is an MCF and shows (1.3).

On the other hand, suppose that $M_{t}$ is an MCF. A computation shows that

$$
(-t)^{\frac{3}{2}} \partial_{t}\left(\frac{x}{\sqrt{-t}}\right)=-t \partial_{t} x+\frac{x}{2} .
$$

If $\frac{M_{t}}{\sqrt{-t}}=M_{-1}$, then

$$
0=(-t)^{\frac{3}{2}}\left\langle\partial_{t}\left(\frac{x}{\sqrt{-t}}\right), \boldsymbol{n}_{M_{-1}}\right\rangle=-t\left\langle\partial_{t} x, \boldsymbol{n}_{M_{-1}}\right\rangle+\frac{1}{2}\left\langle x, \boldsymbol{n}_{M_{-1}}\right\rangle .
$$

Hence, since $M_{t}$ is an MCF, it follows that

$$
H_{M_{-1}}=-\left\langle\partial_{t} x, \boldsymbol{n}_{M_{-1}}\right\rangle=\frac{\left\langle x, \boldsymbol{n}_{M_{-1}}\right\rangle}{2} .
$$

The equation for $H_{M_{t}}$ for general $t$ follows by scaling.

Finally, if an MCF $M_{t}$ satisfies (1.3), then, by the first part of the lemma, $N_{t}=$ $\sqrt{-t} M_{-1}$ is an MCF with the same initial condition as $M_{t}$; thus $M_{t}=N_{t}$ for $t \geq-1$.

The next lemma, which is due to Huisken, [H3] (cf. Ilmanen, page 6 of [I2], [A]; see also [CM1]), computes the first variation of the $F$ functional; since it is so short, we include the proof here. The equivalence of both (2) and (3) with (0.1) follows from this lemma.

Lemma 1.7. If $x^{\prime}=f \boldsymbol{n}$ is a compactly supported normal variation of a hypersurface $\Sigma \subset \mathbb{R}^{n+1}$ and $s$ is the variation parameter, then $\frac{\partial}{\partial s} F\left(\Sigma_{s}\right)$ is

$$
(4 \pi)^{-\frac{n}{2}} \int_{\Sigma} f\left(H-\frac{\langle x, \boldsymbol{n}\rangle}{2}\right) d \mu .
$$


Proof. The first variation formula (for volume) gives

$$
(d \mu)^{\prime}=f H d \mu .
$$

The $s$ derivative of $\log \left[(4 \pi)^{-\frac{n}{2}} \mathrm{e}^{-\frac{|x|^{2}}{4}}\right]$ is given by $-\frac{f}{2}\langle x, \boldsymbol{n}\rangle$. Combining this with (1.9) gives (1.8).

1.2. Self-shrinkers as minimal surfaces. We saw that self-shrinkers in $\mathbb{R}^{n+1}$ are minimal hypersurfaces for the conformally changed metric

$$
g_{i j}=\mathrm{e}^{-\frac{|x|^{2}}{2 n}} \delta_{i j} .
$$

We will use this in the next section to get local estimates and singular compactness, but first investigate these metrics a bit. In particular, we will see that these metrics cannot be made complete and, thus, the compactness of the space of self-shrinkers does not follow from compactness results for minimal surfaces such as the ChoiSchoen, [CS], compactness for positive Ricci curvature; cf. [CM2]. In fact, it turns out the Ricci curvature of these metrics does not have a sign and goes to negative infinity at infinity.

We begin with the obvious observation that the distance to infinity is finite since $\int_{0}^{\infty} \mathrm{e}^{-\frac{t^{2}}{4 n}} d t<\infty$. Furthermore, for $n \geq 2$, the scalar curvature $\widetilde{R}$ of the metric $u^{\frac{4}{n-1}} \delta_{i j}$ is given by ${ }^{5}$

$$
\widetilde{R}=\frac{-4 n}{n-1} u^{\frac{-(n+3)}{n-1}} \Delta u
$$

Thus, for our conformal metrics, we have $u=\mathrm{e}^{\frac{(1-n)|x|^{2}}{8 n}}$. Using that $\Delta \mathrm{e}^{f}=$ $\mathrm{e}^{f}\left(\Delta f+|\nabla f|^{2}\right), \Delta|x|^{2}=2(n+1)$ on $\mathbb{R}^{n+1}$, and $\left.\left.|\nabla| x\right|^{2}\right|^{2}=4|x|^{2}$, we get that

$$
\Delta u=u\left(\frac{(n-1)^{2}}{16 n^{2}}|x|^{2}-\frac{n^{2}-1}{4 n}\right) .
$$

It follows that the scalar curvature is

$$
\widetilde{R}=u^{\frac{-4}{n-1}}\left(n+1-\frac{n-1}{4 n}|x|^{2}\right)=\mathrm{e}^{\frac{|x|^{2}}{2 n}}\left(n+1-\frac{n-1}{4 n}|x|^{2}\right) .
$$

There are a few interesting consequences of this formula. First, the scalar curvature does not have a sign; it is positive when $|x|$ is small and then becomes negative near infinity. Second, as $|x| \rightarrow \infty$, the scalar curvature goes to negative infinity. It follows that the space is not complete; even though infinity is at a finite distance, there is no way to smoothly extend the metric to a neighborhood of infinity.

\footnotetext{
${ }^{5}$ See page 184 in [SY]; the formula there is for an $n$-dimensional manifold, so we have shifted $n$ by one.
} 


\section{Compactness away from a locally finite set of singular points}

We specialize now to self-shrinkers in $\mathbb{R}^{3}$. We will use the following well-known local singular compactness for embedded minimal surfaces in any Riemannian 3-manifold.

Proposition 2.1. Given a point $p$ in a Riemannian 3-manifold $M$. There exists $R>0$ such that the following is true: Suppose $\Sigma_{j}$ are embedded minimal surfaces in $B_{2 R}=B_{2 R}(p) \subset M$ with $\partial \Sigma_{j} \subset \partial B_{2 R}$. If each $\Sigma_{j}$ has area at most $V$ and genus at most $g$ for some fixed $V, g$, then there is a finite collection of points $x_{k}$, a smooth embedded minimal surface $\Sigma \subset B_{R}$ with $\partial \Sigma \subset \partial B_{R}$ and a subsequence of the $\Sigma_{j}$ 's that converges in $B_{R}$ (with finite multiplicity) to $\Sigma$ away from the $x_{k}$ 's.

There are a number of ways to prove this proposition. For instance, one can use the bounds on the area and genus to get uniform total curvature bounds on $B_{3 R / 2} \cap \Sigma_{j}$ (this follows from the local Gauss-Bonnet estimate given in Theorem 3 of [I1]) and then argue as in Choi-Schoen, [CS]. Alternatively, the proposition is an immediate consequence of the much more general compactness results of [CM4]-[CM8] that hold even without the area bound.

Combining Proposition 2.1 with a covering argument (and going to a diagonal subsequence) gives a global singular compactness theorem for self-shrinkers:

Corollary 2.2. Suppose that $\Sigma_{i} \subset \mathbb{R}^{3}$ is a sequence of smooth embedded complete self-shrinkers with genus at most $g, \partial \Sigma_{i}=\emptyset$, and the scale-invariant area bound

$$
\operatorname{Area}\left(B_{R}\left(x_{0}\right) \cap \Sigma_{i}\right) \leq V R^{2}
$$

for all $x_{0} \in \mathbb{R}^{3}$ and all $R>0$. Then there is a subsequence (still denoted by $\Sigma_{i}$ ), a smooth embedded complete (non-trivial) self-shrinker $\Sigma$ without boundary, and a locally finite collection of points $S \subset \Sigma$ so that $\Sigma_{i}$ converges smoothly (possibly with multiplicity) to $\Sigma$ off of $S$.

A set $S \subset \mathbb{R}^{3}$ is said to be locally finite if $B_{R} \cap S$ is finite for every $R>0$.

Proof. The compactness follows by covering $\mathbb{R}^{3}$ by a countable collection of small balls on which we can apply Proposition 2.1 and then passing to a diagonal subsequence. To see that the limit must be non-trivial, observe that every self-shrinker must intersect the closed ball bounded by the spherical self-shrinker. This follows from the maximum principle since the associated MCF's both disappear at the same point in space and time. 


\section{Showing that the convergence is smooth}

It remains to show that the convergence is smooth everywhere. By Allard's theorem, [Al], this follows from showing that the multiplicity must be one. We will show that if the multiplicity is greater than one, then the limit $\Sigma$ is $L$-stable where

$$
L=\Delta+|A|^{2}-\frac{1}{2}\langle x, \nabla(\cdot)\rangle+\frac{1}{2}
$$

is the linearization of the self-shrinker equation (see [CM1]).

Proposition 3.2. If the multiplicity of the convergence of the $\Sigma_{i}$ 's in Corollary 2.2 is greater than one, then $\Sigma$ is L-stable.

The idea for the proof of Proposition 3.2 comes from a related argument for minimal surfaces in [CM9].

Proof of Proposition 3.2. Since the limit surface $\Sigma \subset \mathbb{R}^{3}$ is complete, properly embedded, and has no boundary, $\Sigma$ separates $\mathbb{R}^{3}$ and has a well-defined unit normal $\boldsymbol{n}$. By assumption, the convergence of the $\Sigma_{i}$ 's to $\Sigma$ is not smooth and, thus, by Allard's theorem $[\mathrm{Al}]$ must have multiplicity greater than one.

Existence of a positive solution $\boldsymbol{u}$ of $\boldsymbol{L} \boldsymbol{u}=\mathbf{0}$. Let $\delta$ be the (non-empty) locally finite collection of singular points for the convergence. Since the convergence is smooth away from the $y_{i}$ 's, we can choose $\epsilon_{i} \rightarrow 0$ and domains $\Omega_{i} \subset \Sigma$ exhausting $\Sigma \backslash \delta$ so that each $\Sigma_{i}$ decomposes locally as a collection of graphs over $\Omega_{i}$ and is contained in the $\epsilon_{i}$ tubular neighborhood of $\Sigma$. By embeddedness (and orientability), these sheets are ordered by height. Let $w_{i}^{+}$and $w_{i}^{-}$be the functions representing the top and bottom sheets over $\Omega_{i}$. Arguing as in equation (7) of [Si2], the difference $w_{i}=w_{i}^{+}-w_{i}^{-}$satisfies $L w_{i}=0$ up to higher order correction terms since the operator $L$ given by (3.1) is the linearization of the self-shrinker equation (this is proven in Section 4 in [CM1]).

Fix some $y \notin S$ and set $u_{i}=w_{i} / w_{i}(y)$. Since the $u_{i}$ 's are positive (i.e., the sheets are disjoint), the Harnack inequality implies local $C^{\alpha}$ bounds (Theorem 8.20 of [GiTr]). Elliptic theory then gives $C^{2, \alpha}$ estimates (Theorem 6.2 of [GiTr]). By the Arzelà-Ascoli theorem, a subsequence converges uniformly in $C^{2}$ on compact subsets of $\Sigma \backslash \delta$ to a non-negative function $u$ on $\Sigma \backslash \delta$ which satisfies

$$
L u=0 \quad \text { and } \quad u(y)=1 .
$$

It remains to show that $u$ extends smoothly across the $y_{k}$ 's to a solution of $L u=0$. This follows from standard removable singularity results for elliptic equations once we show that $u$ is bounded up to each $y_{k}$. Consider the cylinder $N_{k}$ (in exponential normal coordinates) over $B_{\epsilon}\left(y_{k}\right) \subset \Sigma$. If $\epsilon$ is sufficiently small, then a result of White 
(see the appendix of [W2]) gives a foliation by minimal (in the conformal metric) graphs $v_{t}$ of some normal neighborhood of $\Sigma$ in $N_{k}$ so that

$$
\begin{aligned}
& v_{0}(x)=0 \text { for all } x \in B_{\epsilon}\left(y_{k}\right), \text { and } \\
& v_{t}(x)=t \text { for all } x \in \partial B_{\epsilon}\left(y_{k}\right) .
\end{aligned}
$$

Furthermore, the Harnack inequality implies that $t / C \leq v_{t} \leq C t$ for some $C>0$. In particular, combining (3.4) with the maximum principle for minimal surfaces (and the Hausdorff convergence of the $\Sigma_{i}$ 's to $\left.\Sigma\right)$, we see that $u_{i}$ is bounded on $B_{\epsilon}\left(y_{k}\right)$ by a multiple of its supremum on $B_{\epsilon}\left(y_{k}\right) \backslash B_{\epsilon / 2}\left(y_{k}\right)$. We conclude that $u$ has a removable singularity at each $y_{i}$ and thus extends to a non-negative solution of $L u=0$ on all of $\Sigma$; since $u(y)=1$, the Harnack inequality implies that $u$ is everywhere positive.

Using $\boldsymbol{u}$ to prove $\boldsymbol{L}$-stability. We will now use a variation on an argument of Fischer-Colbrie-Schoen (see, e.g., Proposition 1.26 in [CM2]). Set $w=\log u$, so that

$$
\Delta w=\frac{\Delta u}{u}-|\nabla w|^{2}=-|A|^{2}+\frac{1}{2}\langle x, \nabla w\rangle-\frac{1}{2}-|\nabla w|^{2} .
$$

Given $\phi$ with compact support, applying Stokes' theorem to div $\left(\phi^{2} \mathrm{e}^{\frac{-|x|^{2}}{4}} \nabla w\right)$ gives

$$
\begin{aligned}
0 & =\int\left(2 \phi\langle\nabla \phi, \nabla w\rangle+\left[-|A|^{2}-\frac{1}{2}-|\nabla w|^{2}\right] \phi^{2}\right) \mathrm{e}^{\frac{-|x|^{2}}{4}} \\
& \leq \int\left(|\nabla \phi|^{2}-|A|^{2} \phi^{2}-\frac{1}{2} \phi^{2}\right) \mathrm{e}^{\frac{-|x|^{2}}{4}}=-\int_{\Sigma}(\phi L \phi) \mathrm{e}^{\frac{-|x|^{2}}{4}},
\end{aligned}
$$

where the inequality used $2 \phi\langle\nabla \phi, \nabla w\rangle \leq \phi^{2}|\nabla w|^{2}+|\nabla \phi|^{2}$ and the last equality came from applying Stokes' theorem to $\operatorname{div}\left(\phi \nabla \phi \mathrm{e}^{\frac{-|x|^{2}}{4}}\right)$.

Proof of Theorem 0.2. We will argue by contradiction. Suppose therefore that there is a sequence of smooth complete embedded self-shrinkers $\Sigma_{i} \subset \mathbb{R}^{3}$ with genus $g$, $\partial \Sigma=\emptyset$, and the scale-invariant area bound

$$
\text { Area }\left(B_{R}\left(x_{0}\right) \cap \Sigma_{i}\right) \leq V R^{2}
$$

for all $x_{0} \in \mathbb{R}^{3}$ and all $R>0$, but so that $\Sigma_{i}$ does not have any smoothly convergent subsequences. By Corollary 2.2, we can pass to a subsequence so that the $\Sigma_{i}$ 's converge (possibly with multiplicity) to an embedded self-shrinker $\Sigma$ away from a locally finite set $S \subset \Sigma$. By assumption, $S$ is non-empty and, by Allard's theorem, the convergence has multiplicity greater than one. Consequently, Proposition 3.2 implies that $\Sigma$ is $L$-stable. However, Theorem 0.5 gives that no such $\Sigma$ exists, giving the desired contradiction. 


\section{A. There are no $L$-stable self-shrinkers}

In this appendix, we will include a proof of Theorem 0.5 from [CM1] for the reader's convenience. Throughout, the smooth complete embedded hypersurface $\Sigma \subset \mathbb{R}^{n+1}$ will be a self-shrinker without boundary and with polynomial volume growth.

We will need the following calculation from [CM1]: The normal part $\langle v, \boldsymbol{n}\rangle$ of a constant vector field $v$ is an eigenfunction of $L$ with

$$
L\langle v, \boldsymbol{n}\rangle=\frac{1}{2}\langle v, \boldsymbol{n}\rangle .
$$

Proof of Theorem 0.5 . We will construct a compactly supported function $u$ that does not satisfy (0.3). Fix a point $p$ in $\Sigma$ and define a function $v$ on $\Sigma$ by

$$
v(x)=\langle\boldsymbol{n}(p), \boldsymbol{n}(x)\rangle .
$$

It follows that $v(p)=1,|v| \leq 1$, and, by (A.1), that $L v=\frac{1}{2} v$. Therefore, given any smooth function $\eta$, we have

$$
\begin{aligned}
L(\eta v) & =\eta L v+v\left(\Delta \eta-\frac{1}{2}\langle x, \nabla \eta\rangle\right)+2\langle\nabla \eta, \nabla v\rangle \\
& =\frac{1}{2} \eta v+v\left(\Delta \eta-\frac{1}{2}\langle x, \nabla \eta\rangle\right)+2\langle\nabla \eta, \nabla v\rangle .
\end{aligned}
$$

Taking $\eta$ to have compact support, we get that

$$
\begin{aligned}
-\int \eta v L(\eta v) \mathrm{e}^{\frac{-|x|^{2}}{4}} & =-\int\left[\frac{1}{2} \eta^{2} v^{2}+\eta v^{2}\left(\Delta \eta-\frac{1}{2}\langle x, \nabla \eta\rangle\right)\right. \\
& \left.+\frac{1}{2}\left\langle\nabla \eta^{2}, \nabla v^{2}\right\rangle\right] \mathrm{e}^{\frac{-|x|^{2}}{4}} \\
& =-\int\left[\frac{1}{2} \eta^{2} v^{2}-v^{2}|\nabla \eta|^{2}\right] \mathrm{e}^{\frac{-|x|^{2}}{4}},
\end{aligned}
$$

where the second equality uses Stokes' theorem to $\frac{1}{2} \operatorname{div}\left(v^{2} \nabla \eta^{2} \mathrm{e}^{\frac{-|x|^{2}}{4}}\right)$ to get

$$
\int \frac{1}{2}\left\langle\nabla \eta^{2}, \nabla v^{2}\right\rangle \mathrm{e}^{\frac{-|x|^{2}}{4}}=-\int v^{2}\left(\eta \Delta \eta+|\nabla \eta|^{2}-\frac{1}{2} \eta\langle x, \nabla \eta\rangle\right) \mathrm{e}^{\frac{-|x|^{2}}{4}} \text {. }
$$

If $\eta$ is identically one on $B_{R}$ and cuts off linearly to zero on $B_{R+1} \backslash B_{R}$, then (A.4) gives

$$
-\int \eta v L(\eta v) \mathrm{e}^{\frac{-|x|^{2}}{4}} \leq \int_{\Sigma \backslash B_{R}} v^{2} \mathrm{e}^{\frac{-|x|^{2}}{4}}-\frac{1}{2} \int_{B_{R} \cap \Sigma} v^{2} \mathrm{e}^{\frac{-|x|^{2}}{4}} .
$$

However, since $|v| \leq 1$ and $\Sigma$ has polynomial volume growth, we know that

$$
\lim _{R \rightarrow \infty} \int_{\Sigma \backslash B_{R}} v^{2} \mathrm{e}^{\frac{-|x|^{2}}{4}}=0
$$


so the right-hand side of (A.7) must be negative for all sufficiently large $R$ 's. In particular, when $R$ is large, the function $u=\eta v$ does not satisfy (0.3). This completes the proof.

\section{References}

[Al] W. K. Allard, On the first variation of a varifold. Ann. of Math. 95 (1972) 417-491. Zbl 0252.49028 MR 0307015

[A] S. Angenent, Shrinking doughnuts. In Nonlinear diffusion equations and their equilibrium states. 3, Progr. Nonlinear Differential Equations Appl. 7, Birkhäuser, Boston 1992, 21-38. Zbl 0762.53028 MR 1167827

[AChI] S. B. Angenent, D. L. Chopp, and T. Ilmanen, A computed example of nonuniqueness of mean curvature flow in $\mathbb{R}^{3}$. Comm. Partial Differential Equations 20 (1995), no. 11-12, 1937-1958. MR 1361726

[Br] K. Brakke, The motion of a surface by its mean curvature. Math. Notes 20, Princeton University Press, Princeton, N.J., 1978. Zbl 0386.53047 MR 0485012

[CS] H. I. Choi and R. Schoen, The space of minimal embeddings of a surface into a three-dimensional manifold of positive Ricci curvature. Invent. Math. 81 (1985), no. 3, 387-394. Zbl 0577.53044 MR 0807063

[Ch] D. Chopp, Computation of self-similar solutions for mean curvature flow. Experiment. Math. 3 (1994), no. 1, 1-15. Zbl 0811.53011 MR 1302814

[CM1] T. H. Colding and W. P. Minicozzi II, Generic mean curvature flow I; generic singularities. Ann. of Math. 175 (2012), 755-833.

[CM2] T. H. Colding and W. P. Minicozzi II, Minimal surfaces. Courant Lect. Notes Math. 4, NYU, Courant Institute of Mathematical Sciences, New York 1999. Zbl 0987.49025 MR 1683966

[CM3] T. H. Colding and W. P. Minicozzi II, Shapes of embedded minimal surfaces. Proc. Natl. Acad. Sci. USA 103 (2006), no. 30, 11106-11111. Zbl 1175.53008 MR 2242650

[CM4] T. H. Colding and W. P. Minicozzi II, The space of embedded minimal surfaces of fixed genus in a 3-manifold. I. Estimates off the axis for disks. Ann. of Math. (2) 160 (2004), no. 1, 27-68. Zbl 1070.53031 MR 2119717

[CM5] T. H. Colding and W. P. Minicozzi II, The space of embedded minimal surfaces of fixed genus in a 3-manifold. II. Multi-valued graphs in disks. Ann. of Math. (2) 160 (2004), no. 1, 69-92. Zbl 1070.53032 MR 2119718

[CM6] T. H. Colding and W. P. Minicozzi II, The space of embedded minimal surfaces of fixed genus in a 3-manifold. III. Planar domains. Ann. of Math. (2) 160 (2004), no. 2, 523-572. Zbl 1076.53068 MR 2123932

[CM7] T. H. Colding and W. P. Minicozzi II, The space of embedded minimal surfaces of fixed genus in a 3-manifold. IV. Locally simply connected. Ann. of Math. (2) 160 (2004), no. 2, 573-615. Zbl 1076.53069 MR 2123933

[CM8] T. H. Colding and W. P. Minicozzi II, The space of embedded minimal surfaces of fixed genus in a 3-manifold. V. Fixed genus. Preprint 2005. arXiv:0509647v1 [math.DG] 
[CM9] T. H. Colding and W. P. Minicozzi II, Embedded minimal surfaces without area bounds in 3-manifolds. In Geometry and topology: Aarhus (1998), Contemp. Math. 258, Amer. Math. Soc., Providence, R.I., 2000, 107-120. Zbl 0999.53006 MR 1778099

[GiTr] D. Gilbarg and N. Trudinger, Elliptic partial differential equations of second order. Grundlehren Math. Wiss. 224, Springer Verlag, Berlin 1983.

Zbl 0562.35001 MR 0737190

[H1] G. Huisken, Flow by mean curvature of convex surfaces into spheres. J. Differential Geom. 20 (1984) no. 1, 237-266. Zbl 0556.53001 MR 0772132

[H2] G. Huisken, Singularity formation in geometric evolution equations. In Workshop on theoretical and numerical aspects of geometric variational problems, Proc. Centre Math. Appl. Austral. Nat. Univ. 26, Australian National University, Centre for Mathematics and its Applications, Canberra 1991, 128-139. Zbl 0747.58024 MR 1139034

[H3] G. Huisken, Asymptotic behavior for singularities of the mean curvature flow. J. Differential Geom. 31 (1990), no. 1, 285-299. Zbl 0694.53005 MR 1030675

[H4] G. Huisken, Local and global behaviour of hypersurfaces moving by mean curvature. In Differential geometry: partial differential equations on manifolds (Los Angeles, CA, 1990), Proc. Sympos. Pure Math. 54, Part 1, Amer. Math. Soc., Providence, R.I., 1993, 175-191. Zbl 0791.58090 MR 1216584

[I1] T. Ilmanen, Singularities of mean curvature flow of surfaces. Preprint, 1995. http://www.math.ethz.ch/ ilmanen/papers/pub.html

[I2] T. Ilmanen, Lectures on mean curvature flow and related equations (Trieste notes), 1995. http://www.math.ethz.ch/ ilmanen/papers/pub.html

[N1] X. H. Nguyen, Construction of complete embedded self-similar surfaces under mean curvature flow. Part I. Trans. Amer. Math. Soc. 361 (2009), no. 4, 1683-1701. Zbl MR

[N2] X.H. Nguyen, Construction of complete embedded self-similar surfaces under mean curvature flow. Adv. Differential Equations 15 (2010), no. 5-6, 503-530. Zbl 1200.53061 MR 2643233

[N3] X. H. Nguyen, Translating tridents. Comm. Partial Differential Equations 34 (2009), no. 3, 257-280. Zbl 1187.53071 MR 2512861

[SY] R. Schoen and S.-T. Yau, Lectures on differential geometry. Conf. Proc. Lecture Notes Geom. Topology 1, International Press, Cambridge, MA, 1994. Zbl 0830.53001 MR 1333601

[Si1] L. M. Simon, Lectures on geometric measure theory. Proc. Centre Math. Anal. Austral. Nat. Univ. 3, Australian National University, Centre for Mathematical Analysis, Canberra 1983. Zbl 0546.49019 MR 0756417

[Si2] L. M. Simon, A strict maximum principle for area minimizing hypersurfaces. J. Differential Geom. 26 (1987), 327-335. Zbl 0625.53052 MR 0906394

[W1] B. White, Evolution of curves and surfaces by mean curvature. In Proceedings of the International Congress of Mathematicians (Beijing, 2002), Vol. I, Higher Education Press, Beijing 2002, 525-538. Zbl 1036.53045 MR 1989203

[W2] B. White, Curvature estimates and compactness theorems in 3-manifolds for surfaces that are stationary for parametric elliptic functionals. Invent. Math. 88 (1987), no. 2, 243-256. Zbl 0615.53044 MR 0880951 
Vol. 87 (2012)

[W3] B. White, The topology of hypersurfaces moving by mean curvature. Comm. Anal. Geom. 3 (1995), no. 1-2, 317-333. Zbl 0858.58047 MR 1362655

Received July 7, 2009

Tobias H. Colding, MIT, Department of Mathematics, 77 Massachusetts Avenue, Cambridge, MA 02139-4307, U.S.A.

E-mail: colding@math.mit.edu

William P. Minicozzi II, Johns Hopkins University, Department of Mathematics, 3400 N. Charles St., Baltimore, MD 21218, U.S.A.

E-mail: minicozz@math.jhu.edu 